\title{
IL-6 Receptor Blockade by Tocilizumab Has Anti-absence and Anti-epileptogenic Effects in the WAG/Rij Rat Model of Absence Epilepsy
}

\author{
Antonio Leo ${ }^{1}$. Valentina Nesci ${ }^{1}$ - Martina Tallarico ${ }^{1}$ - Nicola Amodio ${ }^{2}$ Eugenia M. Gallo Cantafio ${ }^{1}$. \\ Giovambattista De Sarro ${ }^{1} \cdot$ Andrew Constanti $^{3} \cdot$ Emilio Russo $^{1}$ (D) $\cdot$ Rita Citraro $^{1}$
}

Published online: 17 July 2020

(C) The American Society for Experimental NeuroTherapeutics, Inc. 2020

\begin{abstract}
Increased expression of interleukin-6 (IL-6) both in cerebrospinal fluid (CSF) and plasma is closely associated with convulsive epilepsy and symptom severity of depression. By comparison, at present, little is known about the role of this cytokine in childhood (non-convulsive) absence epilepsy. The aim of this work was to investigate the potential effects of acute and chronic treatment with tocilizumab (TCZ, 10 and $30 \mathrm{mg} / \mathrm{kg} /$ day), on absence seizures, their development, and related psychiatric comorbidity in WAG/Rij rats. It is known that lipopolysaccharide (LPS)-induced changes in inflammatory processes increase absence epileptic activity. In order to study the central effects of TCZ, we investigated whether administration of this anti-IL-6R antibody could modulate the lipopolysaccharide (LPS) or IL-6-evoked changes in absence epileptic activity in WAG/Rij rats. Our results demonstrate that TCZ, at both doses, significantly reduced the development of absence seizures in adult WAG/Rij rats at 6 months of age ( 1 month after treatment suspension) compared with untreated controls, thus showing disease-modifying effects. Decreased absence seizure development at 6 months of age was also accompanied by reduced comorbid depressive-like behavior, whereas no effects were observed on anxiety-related behavior. Acute treatment with TCZ, at $30 \mathrm{mg} / \mathrm{kg}$, had antiabsence properties lasting $\sim 25 \mathrm{~h}$. The co-administration TCZ with i.c.v. LPS or IL-6 showed that TCZ inhibited the worsening of absence seizures induced by both proinflammatory agents in the WAG/Rij rats, supporting a central anti-inflammatory-like protective action. These results suggest the possible role of IL- 6 and consequent neuroinflammation in the epileptogenic process underlying the development and maintenance of absence seizures in WAG/Rij rats. Accordingly, IL-6 signaling could be a promising pharmacological target in absence epilepsy and depressive-like comorbidity.
\end{abstract}

Key Words Absence epilepsy · epileptogenesis · neuroinflammation · tocilizumab · depressive-like behavior · anxiety.

\section{Introduction}

Clinical and preclinical data, already in the last century, suggest a potential role of neuroinflammation and immune reactions in the pathophysiology of epilepsy and

Emilio Russo

erusso@unicz.it

1 Science of Health Department, School of Medicine and Surgery, Magna Græcia University of Catanzaro, Viale Europa - Germaneto, 88100 Catanzaro, Italy

2 Experimental and Clinical Medicine Department, School of Medicine and Surgery, Magna Græcia University, Catanzaro, Italy

3 Pharmacology Department, UCL School of Pharmacy, London, UK epileptogenesis [1-3]. Chronic brain inflammation may also contribute to intractability of seizures and their neurobehavioral comorbidities $[4,5]$. Among the vast array of inflammatory mediators contributing to the complexity of neuroinflammation, particular attention has been devoted to cytokines such as interleukin (IL)-1 $\beta$ and IL-6 and tumor necrosis factor (TNF) $[6,7]$.

Inflammatory cytokines are known to increase the excitability of neurons and dysregulate the neural connectivity that underlies epileptogenesis [7-9]. Changes in cytokine expression also occur following seizures and seizures can be aggravated when cytokines are added exogenously [9]. Alteration of proinflammatory cytokines is also associated with several psychiatric disorders $[10,11]$ with the latter being often considered an 
epilepsy comorbidity with depression being the most common [5, 12]; however, a bidirectional link should be considered [13].

IL-6 is a pleiotropic cytokine that exerts its actions via the classic signaling pathway when it binds to the membranebound IL-6R or via the trans-signaling pathway upon binding to the soluble form of IL-6R (sIL-6R); IL-6 is primarily secreted by activated astrocytes and microglia. Classic IL-6 signaling is responsible for the anti-inflammatory properties of IL-6, whereas trans-signaling is responsible for its proinflammatory actions [14]. Dysregulation of the IL-6 axis can lead to the onset or development of different disorders including CNS pathologies $[15,16]$.

Based on its major influence on the production of inflammatory factors, IL- 6 can be considered one of the promoters of the inflammatory response in the brain, together with TNF- $\alpha$ and IL- $1 \beta[15,17]$. Studies in both preclinical and clinical epilepsy have found an increased expression of IL-6 both in brain and blood that is closely associated with convulsive seizures [18-21]. Similarly, different evidence also indicates that both peripheral and central IL-6 levels are significantly elevated in depressive disorders [22].

In contrast, little is known about the exact role of inflammation/proinflammatory cytokines in the genesis and/ or modulation of absence epileptic activity in WAG/Rij rats, an animal model of absence epilepsy and epileptogenesis with neuropsychiatric comorbidities [23-25]. Some studies suggested that inflammatory processes and proinflammatory cytokines may have a role in the genesis/modulation of spontaneous absence epileptic activity in WAG/Rij rats [26, 27]. Systemic or central administration of bacterial endotoxin lipopolysaccharide (LPS) in rodents activates microglia and increases expression of many brain cytokines (including IL$1 \beta$, TNF- $\alpha$, and IL-6) changing the seizure threshold and enhancing epileptogenesis [28, 29].

It has also been shown that the LPS-evoked increase in proinflammatory cytokines can increase absence epileptic activity in some animal models $[30,31]$. It is known that IL- $1 \beta$ increases absence seizures, and TNF- $\alpha$ delays the onset of spontaneous spike-wave discharges (SWDs) [26] but it remains unknown whether IL-6 modulates the occurrence of SWDs. Considering the strong evidence linking IL-6 to pathophysiology, clinical expression, and treatment response in epilepsy and depression, we hypothesized that a treatment that blocks or reduces this proinflammatory cytokine production and/or action could have a therapeutic effect in absence seizures and psychiatric comorbidity. IL-6 blockade is a current therapeutic strategy for autoimmune/inflammatory conditions [32], and a possible anti-epileptogenic/epileptic and antidepressant effect of IL-6 antagonism can be also postulated as potentially useful in these disorders. Tocilizumab (TCZ), a humanized monoclonal antibody against the IL-6 receptor, is currently used for the treatment of IL-6-induced immune diseases, including rheumatoid arthritis [32], and the successful treatment of refractory status epilepticus with TCZ has also recently been reported $[33,34]$. In the present work, we studied the effects of TCZ on established SWD seizures and epileptogenesis in WAG/Rij rats, and also investigated whether TCZ could influence both depressive- and anxiety-like comorbidities in this model.

\section{Materials and Methods}

\section{Animals}

Male WAG/Rij rats $(n=111)$, purchased from Charles River Laboratories s.r.l. (Calco, Lecco, Italia), were used in all experiments. Animals were housed three/four per cage and kept under controlled environmental conditions $(60 \pm 5 \%$ humidity; $22 \pm 2{ }^{\circ} \mathrm{C} ; 12 / 12 \mathrm{~h}$ reversed light/dark cycle; lights on at 8:00 p.m.) with free access to standard laboratory chow and tap water. Animal care and experimental procedures were conducted in conformity with the international and national laws and policies and after approval by our local ethical committee (EU Directive 2010/63/EU for animal experiments, ARRIVE guidelines, and the Basel declaration including the 3R concept). The experimental protocols and the procedures reported herein were approved (Authorization $n^{\circ}$ 491/2016PR) by the Animal Care Committee of the University of Catanzaro, Italy. All efforts were made to minimize animal suffering and to use only the number of animals necessary to produce reliable scientific data.

\section{Study Design}

The goals of the present study were to evaluate, in WAG/Rij rats, the effects of TCZ (RoActemra $20 \mathrm{mg} / \mathrm{ml}$ concentrate for solution for infusion, Roche, Italy) on:

1) Established absence seizures (acute treatment);

2) The epileptogenic process underlying the development of absence seizures (early long-term treatment; ELTT);

3) Symptoms of depressive-like behavior and anxiety in ELTT rats;

4) IL-6 levels in serum and in the brain;

5) The LPS or IL-6-dependent increase in seizure parameters.

The doses of TCZ (10 and $30 \mathrm{mg} / \mathrm{kg}$ ) were obtained starting from the commercial product in which TCZ $(20 \mathrm{mg} /$ $\mathrm{mL}$ ) is solubilized in sterile water added of an unknown quantity of solutes (i.e., L-histidine, L-histidine monohydrochloride monohydrate, L-arginine hydrochloride, L-methionine, polysorbate 80 ) and injected at the desired dose. Lacking the 
possibility of exactly replicate the same vehicle, control animals were injected with saline solution $(0.9 \% \mathrm{NaCl})$.

\section{Acute TCZ Treatment}

Adult WAG/Rij rats ( $\sim 6$ months old; $n=18)$ were administered intraperitoneally (i.p.) with two doses of TCZ (10 and $30 \mathrm{mg} / \mathrm{kg}$; i.p.) in order to determine if this would significantly influence the number and duration of SWDs. Separate groups of rats $(n=6$ for each dose) were used to determine the effects of vehicle (saline) and TCZ. Since TCZ has an extended half-life (between 6 and 9 days in rats and $\sim 10$ days in humans) $[35,36]$, EEG effects were studied for 4 consecutive days. Each recording session lasted for $3 \mathrm{~h}$ and started, the first day, $1 \mathrm{~h}$ after the administration of TCZ or vehicle (saline solution). Therefore, the recording session of the first day consisted in $1 \mathrm{~h}$ of baseline EEG signals without injection, and $3 \mathrm{~h}$ after the administration of TCZ or vehicle (saline solution). The other 3 days of EEG recordings consisted of $3 \mathrm{~h}$ without administration (start at 9:00 a.m.). To assess the pharmacological effects of the TCZ over time, each $3 \mathrm{~h}$ EEG recording was divided into $30 \mathrm{~min}$, and the cumulated SWD number and duration per epoch after drug administration were calculated [37]. A standardized surgery protocol was followed, as previously reported [37].

\section{Early Long-term Treatment with TCZ}

WAG/Rij rats were early long-term treated with TCZ $(n=21$ for each dose) for about 17 weeks (up to the age of 5 months) starting at postnatal day 30 (P30). TCZ was given at 10 and $30 \mathrm{mg} / \mathrm{kg}$ intraperitoneally (i.p.) once a week (every Monday for the 17 weeks of treatment) according to its long-lasting half-life in rats. Age-matched control (vehicle) rats $(n=21)$ were kept under the same housing conditions over the same period of time with vehicle (saline solution). During the treatment period, all animals were weighed weekly every Monday between 9:00 and 11:00 a.m. and particular attention was paid to the possible appearance of any drug-induced side-effects.

After the end of treatment ( -35 months), WAG/Rij rats were normally housed up to the age of $\sim 6$ months when they were subjected to surgery, as previously described [38]. After surgery, WAG/Rij rats from each group (treated and untreated; $n=8$ for each dose and vehicle) were experimentally evaluated by $3 \mathrm{~h}$ of EEG recordings over 3 consecutive days, within 30 and 36 days after treatment discontinuation. The same rats were again EEG recorded at the age of $\sim 10$ months (5 months after treatment discontinuation) to evaluate the duration of TCZ effects [38].

\section{Behavioral Tests in ELTT Rats}

In order to evaluate the ELTT effects of TCZ on depressiveand anxiety-like behavior in WAG/Rij rats, behavioral tests were performed, respectively at $\sim 1$ and $\sim 5$ months after the end of TCZ treatment on a different group of 24 rats $(n=8$ in each group).

Forced swimming test (FST) was performed to assess the duration of immobility time (IT), which is the experimental analog of a depressive-like behavior, whereas the elevated plus maze (EPM) test was used to measure anxiety-related behavior. When two tests were carried out on the same rat, at least 1 day (range 1-3) was allowed, as previously reported $[25,39]$. All behavioral tests were performed under controlled temperature, humidity, and light intensity (dim illumination), and with the support of video-tracking software (EthoVision XT8; Noldus Information Technology, Wageningen, the Netherlands). Experiments were always performed between 09:00 and 11:00 a.m. in order to avoid possible circadian alterations of test results.

\section{Forced Swimming Test}

The FST apparatus consisted of a clear plastic cylinder $47 \mathrm{~cm}$ in height with a $38-\mathrm{cm}$ inside diameter containing $38 \mathrm{~cm}$ of water at $22-23{ }^{\circ} \mathrm{C}$. Rats were individually forced to swim in a plastic cylinder for $6 \mathrm{~min}$. The duration of immobility (immobility time; IT) including passive swimming, was measured during the last $4 \mathrm{~min}$ of the 6 -min testing period. The criterion for passive swimming was floating vertically in the water while making only those movements necessary to keep the head above the water. Increased IT in the FST was indicative of depression-like behavior [25].

\section{Elevated Plus Maze Test}

The EPM test was performed as previously described for WAG/Rij rats [40]. As anxiety-related variables, the time spent and numbers of entries into the open and closed arms were measured. The EPM consisted of a black wooden platform with two open arms $(50 \mathrm{~cm} \times 10 \mathrm{~cm})$ and two closed arms of the same size arranged in the shape of a plus sign. The maze was elevated $80 \mathrm{~cm}$ above the floor and exposed to dim illumination $(40 \mathrm{~W})$. The rats were individually placed in a central platform facing a closed arm and allowed to explore the maze freely for $10 \mathrm{~min}$. The shorter the time spent in the open arms and in the central platform, the higher the anxiety is and vice versa. Mean velocity and total distance moved were also measured and examined for every experimental group. In order to eliminate olfactory cues, the maze was systematically cleaned [40]. 


\section{LPS or IL-6 Co-administration with TCZ Protocol}

Six-month-old WAG/Rij rats were used in this set experiments. Animals were assigned into 5 groups and treated as follows:

Group 1: $(n=6)$ : control rats, non-treated;

Group 2: $(n=6)$ : rats treated with IL-6 $(40 \mathrm{pg} / 2 \mu$ i.c.v.);

Group 3: $(n=6)$ : rats treated with TCZ $(30 \mathrm{mg} / \mathrm{kg}$; i.p.) and IL-6 (40 pg/2 $\mu$ l i.c.v.);

Group 4: $(n=6)$ : rats treated with LPS $(0.5 \mu \mathrm{g} / 5 \mu \mathrm{l}$ i.c.v.);

Group 5: $(n=6)$ : rats treated with TCZ $(30 \mathrm{mg} / \mathrm{kg}$; i.p.) and LPS $(0.5 \mu \mathrm{g} / 5 \mu$ i.c.v. $)$.

For the co-administration, rats were injected, after $1 \mathrm{~h}$ of EEG baseline, with TCZ (30 mg/kg i.p.) followed by IL-6 ( $40 \mathrm{pg} / 2 \mu \mathrm{l}$ i.c.v.) or LPS $(0.5 \mu \mathrm{g} / 5 \mu \mathrm{l}$ i.c.v.) injected $2 \mathrm{~h}$ later. The EEG was recorded for $6 \mathrm{~h}$ ( $2 \mathrm{~h}$ after the TCZ injection and $3 \mathrm{~h}$ after IL-6 or LPS injection), as previously described [41].

\section{Western Blotting Analysis}

Rats were decapitated, and the brains (without cerebellum) were quickly removed and submerged in ice-cold artificial cerebrospinal fluid. The brain tissue was homogenized using the Gentle MACS dissociator (Miltenyi Biotech, Bergisch Gladbach, Germany) in ice-cold NP40 lysis buffer (Life Technologies) containing a cocktail of protease and phosphatase inhibitors (Life Technologies, Monza, Italy) and then centrifuged at $13,362 \mathrm{rcf}$ for $30 \mathrm{~min}$ at $4{ }^{\circ} \mathrm{C}$ to remove tissue debris [40]. Protein lysates $(50 \mu \mathrm{g})$ were electrophoresed through a NuPAGE $4 \%$ to $12 \%$ gel (Life Technologies) and electroblotted onto a nitrocellulose membrane (Life Technologies). The membrane was blocked for $1 \mathrm{~h}$ with $5 \%$ nonfat dry milk/phosphate-buffered saline-Tween $0.05 \%$ (Biorad, Hercules, CA, USA), and then incubated over night with the antibodies for phosphorylated signal transducer and activator of transcription 3 (p-STAT3; Y705) and STAT3 (Cell Signaling, Danvers, MA, USA). The levels of proteins and phosphoproteins were detected with horseradish peroxidase-linked secondary antibodies and the enhanced chemiluminescence system (GE Healthcare, Milan, Italy).

\section{Statistical Analysis}

All statistical procedures were performed using GraphPad Prism 8.0 software (GraphPad Software Inc., La Jolla, CA, USA). EEG recordings were subdivided into 30-min epochs, and the duration and number of SWDs were evaluated separately for every epoch. Such values were averaged, and data obtained were expressed as mean \pm S.E.M. for every compound or drug combination. All experimental data were analyzed and compared by one-way analysis of variance (ANOVA) followed by Dunnett's post hoc test. $p<0.05$ was considered significant.

\section{Results}

\section{Effects of Acute TCZ Administration on Established Absence Seizures}

Acute TCZ administration ( $30 \mathrm{mg} / \mathrm{kg}$; i.p.) in adult WAG/Rij rats with established absence seizures induced a significant decrease $(p<0.05)$ in the number and duration of SWDs. This effect appeared $60 \mathrm{~min}$ after administration, reached a peak at $150 \mathrm{~min}(\sim 50 \%$ reduction; $p=0.039$ for nSWDs and $p=0.041$ for dSWDs) and lasted until the second day of EEG recording, returning to baseline levels the third day of recording. In contrast, the dose of $10 \mathrm{mg} / \mathrm{kg} \mathrm{TCZ}$ produced no significant effects on SWD activity at any time point (Fig. 1A and B). sSWDs were not significantly modified by TCZ treatment $(P=0.13$; data not shown).

\section{Effects of TCZ ELTT on Development of Absence Seizures}

The EEG recording analysis obtained from untreated 6month-old control WAG/Rij rats ( $n=8$ rats) showed a mean number of SWDs (nSWDs) of $10.56 \pm 1.11$, with a mean total duration (dSWDs) of $71.95 \pm 13.14 \mathrm{~s}$, and a mean single duration (sSWD) of $6.41 \pm 0.66$ for a 30 -min epoch. ELTT with TCZ, at both doses, significantly reduced the development of absence seizures in adult WAG/Rij rats at 6 months of age (1 month after treatment suspension) compared with untreated controls showing comparable anti-epileptogenic effects. In details, TCZ at the dose of $10 \mathrm{mg} / \mathrm{kg} /$ week decreased the mean nSWD and dSWD by $\sim 30 \%$ and $43 \%$, respectively $(p<$ 0.005 ), whereas TCZ at the dose of $30 \mathrm{mg} / \mathrm{kg} /$ week decreased the mean nSWD and dSWD by $\sim 37 \%(p=0.002)$ and $48 \%$ $(p=0.0071)$ respectively (Fig. 2$)$. The mean sSWD value was not modified in any group. When the same animals were recorded at 10 months of age ( 5 months after the end of TCZ treatment), the TCZ effects on SWD parameters disappeared returning to control values suggesting only a temporary antiepileptic effect (Fig. 2). Animal growth, over the 17 weeks of treatment, did not significantly differ between TCZ-treated and untreated rats (data not shown).

In order to test the mechanism of TCZ potential effects on seizure development, the brain obtained from 5 animals in each group of control and under TCZ treatment were analyzed at 3 months of treatment ( 4 months of age) and 1 month after drug withdrawal (in concomitance with EEG recordings) by WB analysis. These experiments, unfortunately, did not show any difference of IL-6R downstream signaling, as 
Fig. 1 Effects of acute TCZ administration on absence seizures in WAG/Rij rats. Effects of the administration TCZ at two different doses (10 and $30 \mathrm{mg} / \mathrm{kg}$; i.p.) on the number (A) and duration (B) of characteristic SWDs in 6-month-old WAG/Rij rats (4 days EEG recording). After a single drug injection, EEG data of 4 recording days were separated into epochs of $30 \mathrm{~min}$. A significant reduction of the incidence of SWDs was found during the first day of recording with a peak at 150 min and until the second EEG recording day. Values are means \pm S.E.M of 6 animals for each dose. ${ }^{*} p<0.05$ (one-way ANOVA) vs. the corresponding value obtained in control rats. CTRL = control; i.p. $=$ intraperitoneal; $\mathrm{TCZ}=$ tocilizumab; SWDs = spike--wave discharges
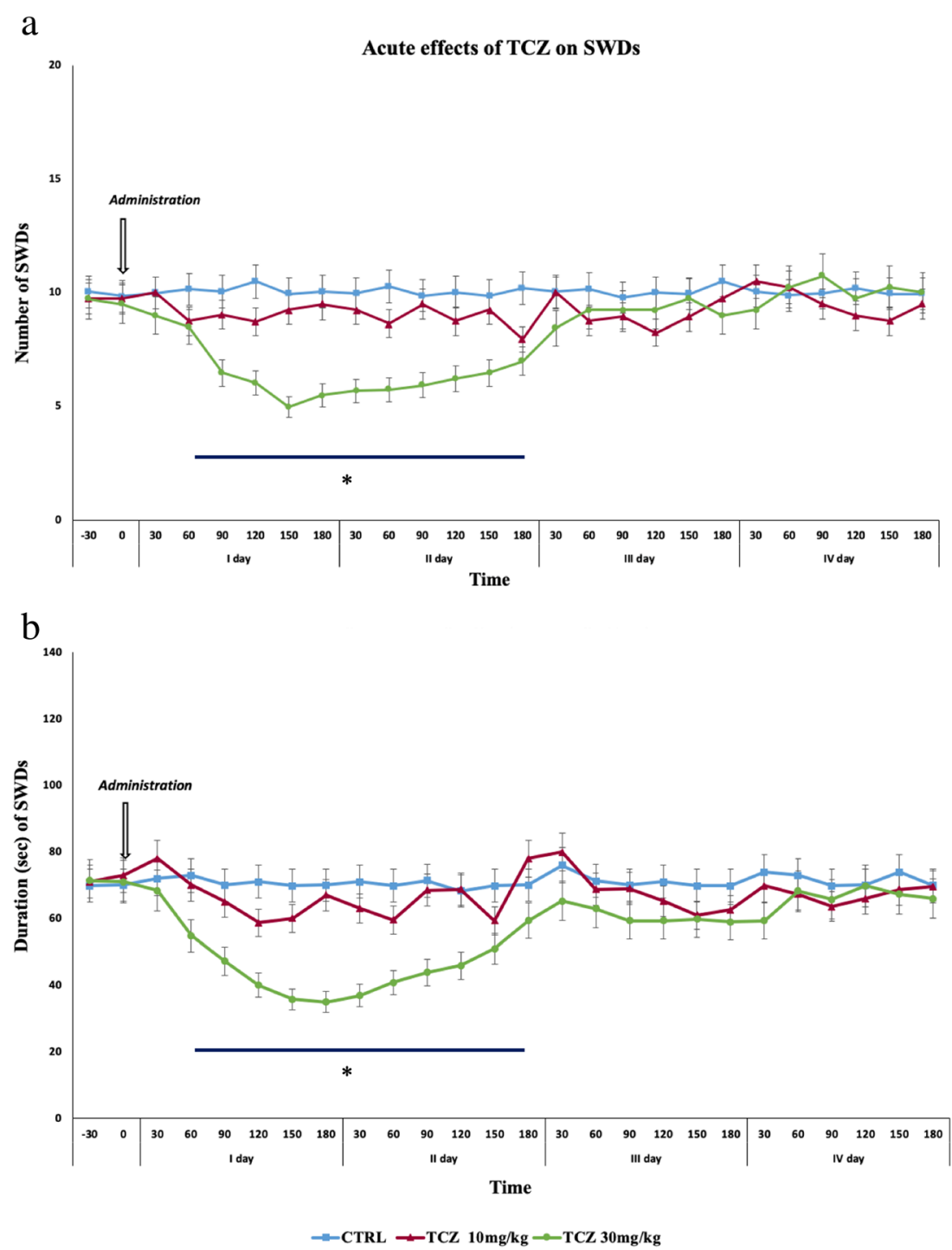

demonstrated by the lack of STAT3 phosphorylation modifications in brains of treated vs no-treated WAG/Rij rats (data not shown).

\section{Effects of ELTT with TCZ on Behavior in WAG/Rij Rats}

We also studied the effects of ELTT with TCZ on anxiety and depressive-like behavior in the WAG/Rij rats at two different ages ( 6 and 10 months old). Control WAG/Rij rats at the age of 6 months display a depressive-like behavior highlighted by an increased IT in the FST, which is linked to the development of SWDs [25].

ELTT with TCZ, at both doses, significantly (CTRL vs TCZ $30 \mathrm{mg} / \mathrm{kg} p=0.0001$; CTRL vs TCZ $10 \mathrm{mg} / \mathrm{kg} p=$ 0.001 ) decreased the IT in 6-month-old WAG/Rij rats (Fig. 3; 1 month after treatment discontinuation), whereas this TCZ antidepressant-like effect was not observed in WAG/Rij rats at 10 months of age ( 5 months after treatment withdrawal)
(Fig. 3; $p>0.05$ ). Mean velocity did not significantly differ (speed about $5 \mathrm{~cm} / \mathrm{s} ; p>0.05$ ) between groups. No effects were observed on anxiety-related behavior, evaluated by EPM, both at 6 and 10 months of age (data not shown).

\section{Changes in SWD Parameters After LPS or IL-6 Administration and TCZ}

LPS administration $(0.5 \mu \mathrm{g} / 5 \mu$ l; i.c.v. $)$ significantly increased the number of SWDs in WAG/Rij rats in accordance with other studies (Fig. 4A) [41]. The number of SWDs was more than doubled ( $\sim 65 \%$ increase) at 150 min after LPS injection and significantly increased between 30 and 270 min after injection. Similarly, an increased duration of SWDs also occurred in the same time interval. The administration of TCZ $(30 \mathrm{mg} / \mathrm{kg}$; i.p.) $120 \mathrm{~min}$ before the LPS injection $(0.5 \mu \mathrm{g} / 5 \mu \mathrm{l}$; i.c.v. $)$ almost fully abolished the effect of LPS on SWD number 


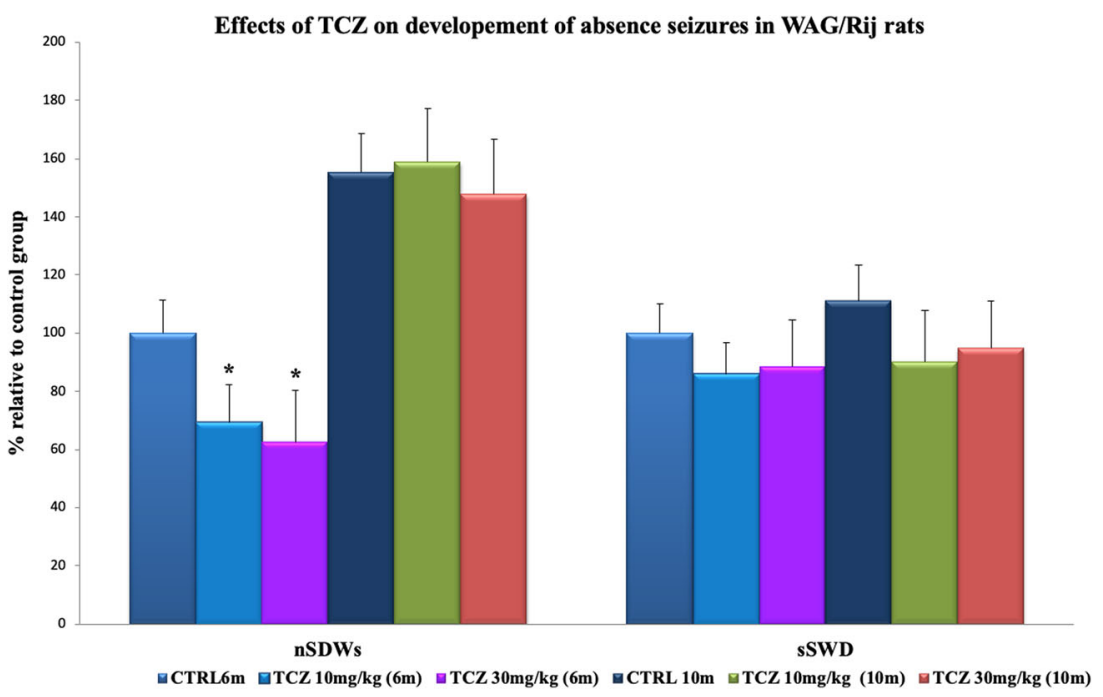

Fig. 2 Effects of ELTT with TCZ on the development of absence seizures in WAG/Rij rats. Effects of chronic treatment (starting at P30 and lasting 17 weeks) with TCZ (10 and $30 \mathrm{mg} / \mathrm{kg} /$ day; i.p.) on SWDs recorded in WAG/Rij rats at 6 and 10 months of age. Data (means \pm SEM, $n=8$ per group) are expressed as percentage change relative to 6-month-old CTRL rats (dotted line; values for control rats were as follows: $\mathrm{nSWDs}=10.56$

and duration (Fig. 4A). LPS was injected at the time of plateau effect of TCZ as demonstrated above (see "Effects of Acute TCZ Administration on Established Absence Seizures") and at the peak of LPS action under TCZ a $25 \%$ increase in SWDs number was observed vs $65 \%$ increase observed without TCZ administration (LPS effects in the two groups were compared by $t$ test of percentage differences relatively to measures of the last epoch before LPS administration; $p<0.05)$. $\pm 1.11 ; \mathrm{dSWDs}=71.95 \pm 13.14 ; \mathrm{nSWDs}=6.41 \pm 0.66)$. $*$ Significantly different $(p<0.05)$ from age-matched CTRL rats. CTRL $=$ control rats; $\mathrm{TCZ}=$ tocilizumab; $\mathrm{nSWDs}=$ mean number of SWDs for every $30-\mathrm{min}$ epoch; dSWDs = mean cumulative duration of SWDs for every 30-min epoch expressed in seconds; $\mathrm{SSWD}=$ mean duration of a single SWD expressed in seconds

IL-6 (40 pg/2 $\mu$ l; i.c.v.) administered alone, increased the SWDs number and duration significantly in the first $4 \mathrm{~h}$ of the EEG recordings with a maximum increase of about $50 \%$ between 60 and $150 \mathrm{~min}$ after injection. The effect of TCZ (30 mg/kg; i.p.) on the IL-6 i.c.v. administration was similar to that of combined TCZ and LPS administration. In particular, pretreatment with TCZ 120 min before the IL-6 injection, almost completely abolished the IL-6-induced SWD parameters increase (Fig. 4B); at the peak of IL-6 action under TCZ a

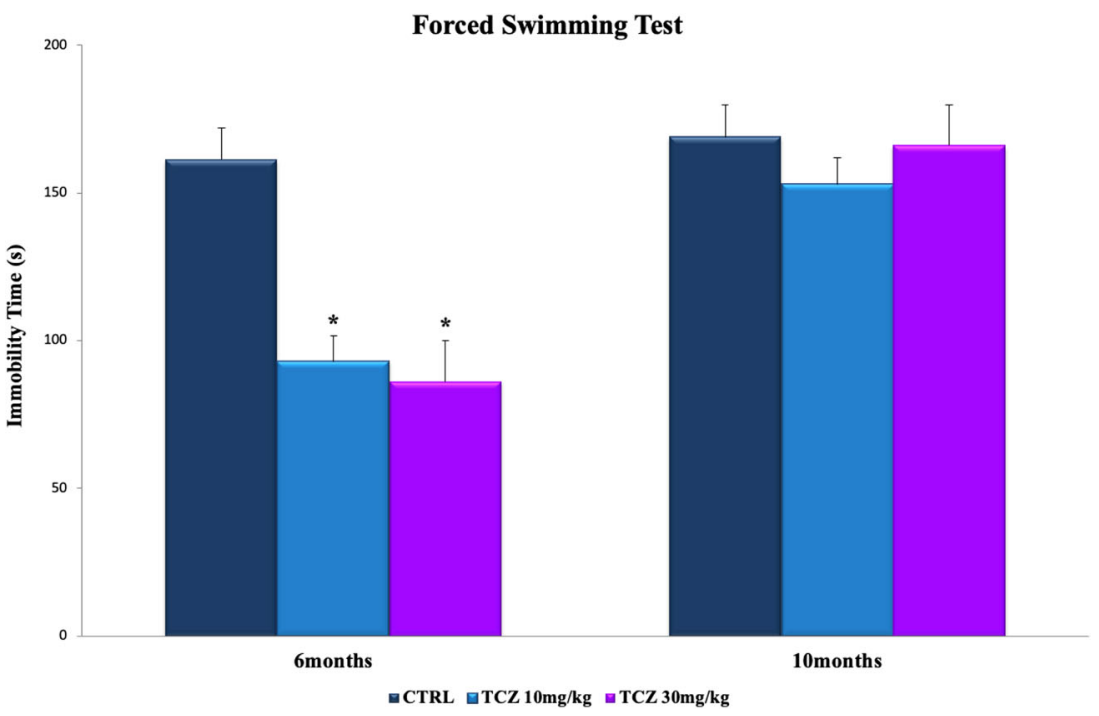

Fig. 3 Effects of ELTT with TCZ on IT in the FST in WAG/Rij rats Effects of chronic treatment (starting at P30 and lasting 17 weeks) with TCZ (10 and $30 \mathrm{mg} / \mathrm{kg} /$ day; i.p.) on IT in the FST in WAG/Rij rats at the age of 6 months (left side) and 10 months (right side). Bars indicate IT expressed in seconds. Values are means \pm SEM of IT (s) $(n=8$ for every group). Data marked with an asterisk are significantly different $(p<0.05)$ from the respective control group $(\mathrm{CTRL})$. IT $=$ immobility time; $\mathrm{FST}=$ forced swimming test; $\mathrm{CTRL}=$ control rats; $\mathrm{TCZ}=$ tocilizumab 
a

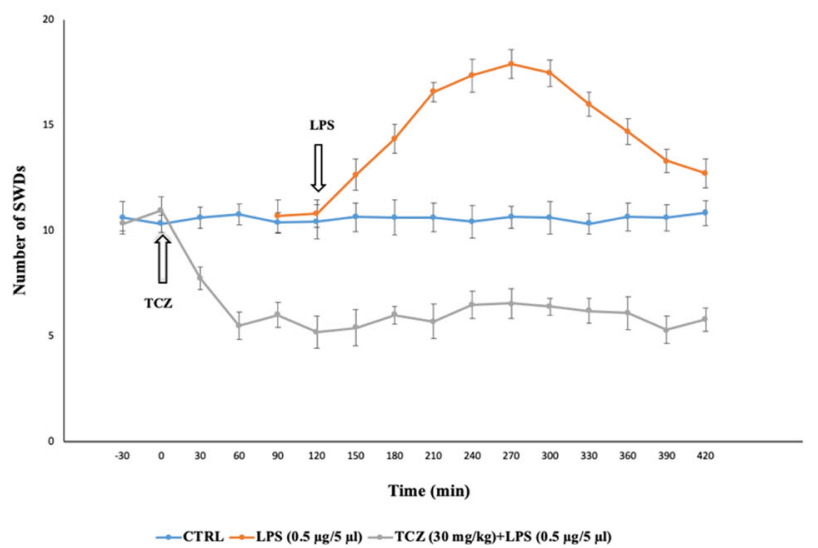

Fig. 4 Effects of acute systemic administration of TCZ $120 \mathrm{~min}$ before LPS or IL-6 administration in adult WAG/Rij rats. (A) Plots show effects of LPS $(0.5 \mu \mathrm{g} / 5 \mu 1$ i.c.v.) alone or in combination with TCZ $(30 \mathrm{mg} / \mathrm{kg}$; i.p.) on the number of epileptic SWDs. (B) Plots show effects of IL-6 (40 pg/2 $\mu$ l; i.c.v.) alone or in combination with TCZ (30 mg/kg; i.p.) on

$18 \%$ increase in SWD number was observed vs $52 \%$ increase observed without TCZ administration (IL-6 effects in the two groups were compared by $t$ test of percentage differences relatively to measures of the last epoch before IL-6 administration; $p<0.05)$.

\section{Discussion}

Inflammatory cytokines such as IL-6 significantly contribute to the generation and recurrence of spontaneous epileptic seizures and to neurological comorbidities in animal epilepsy models [5, 42]. IL-6 is a critical cytokine controlling the transition from innate to acquired immunity, which is responsible for damage to brain tissue, and where IL-6 trans-signaling is very important. This suggests a functional role for IL-6 in epilepsy and psychiatric comorbidity and a potential target for treating these disorders in humans [17].

The present study revealed some interesting new findings in general accordance with the above proepileptic role of IL-6. Firstly, early chronic treatment with the IL-6R blocker TCZ reduced the epileptogenesis process (i.e., disease modifying or delaying epilepsy development) and decreased depressive behavioral comorbidity in the genetic WAG/Rij rat model of absence epilepsy. Secondly, acute TCZ treatment had antiepileptic effects on SWD parameters in adult WAG/Rij rats, supporting a role for neuroinflammation in this model. Thirdly, TCZ/LPS or TCZ/IL-6 co-administration studies showed that TCZ prevented the LPS or IL-6-dependent increases in absence seizures, further indicating that TCZ effects are mediated by IL- 6 that plays a role in epileptogenic process also in this model. b

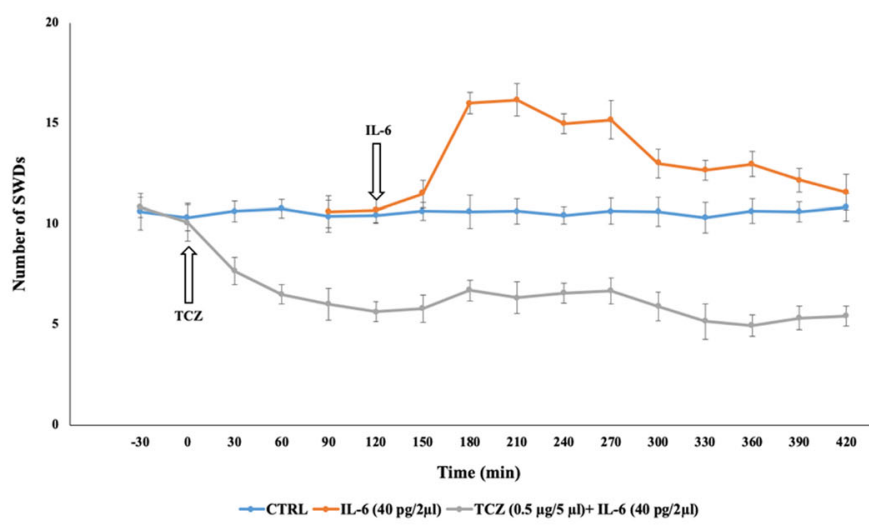

the number of epileptic SWDs. Data values are means \pm SEM $(n=8$ for each compound or drug combination). LPS = lipopolysaccharide; IL-6 = Interleukin 6; $\mathrm{TCZ}=$ Tocilizumab; i.c.v. $=$ intracerebroventricular; i.p. $=$ intraperitoneal

\section{Tocilizumab and Epilepsy/Epileptogenesis}

Our data have demonstrated for the first time that a continuous treatment with TCZ, started prior to the onset of seizures, reduces the subsequent development of EEG absence epilepsy in adult WAG/Rij rats, thereby displaying anti-epileptogenic properties. However, this effect found at 6 months of age was only temporary, since at 5 months after drug withdrawal, absence seizures returned to their control levels. This latter result may indicate that full development of absence seizures is possibly delayed and not necessarily disease modifying. Furthermore, we demonstrated that acutely administered TCZ in WAG/Rij rats at the age of 6 months (with absence seizures already established) was also able to reduce both the number and total duration of SWDs up to $50 \%$ as compared to untreated control rats, exhibiting direct antiseizure properties. However, no direct dose response effect was observed indicating that a ceiling effect has been reached similarly to what was observed also with other drugs acting on inflammation in this model (e.g., etoricoxib, indomethacin) [43, 44] but also by levetiracetam [45]. In accordance with our results, recent data have indicated that after blocking IL-6Rs also by $\mathrm{TCZ}$, a reduction of epileptic seizures in experimental and human refractory epilepsy can be achieved $[33,34,46]$.

The role of IL-6 in WAG/Rij rats and absence epilepsy has not been directly investigated, while the involvement of neuroinflammation has only been poorly studied [23, 26, 27]. In an attempt to determine whether IL-6 pathway was hyperactivated into the brain of WAG/Rij rats, we measured the phosphorylation levels of STAT3 (which is downstream activated following IL-6R activation) but found no differences when comparing TCZ-treated and untreated groups. A possible explanation is due to the fact that we attempted to measure 
phospho-STAT3 in the full brain. In fact, it is possible that an increase in IL-6 levels occurs in specific brain areas that we have not been able to measure. Interestingly, previous studies showed that alterations in proinflammatory cytokines are associated with the location of the epileptogenic zone [47]. Accordingly, IL-1 $\beta$ was only found to be modified in the somatosensory cortex of GAERS (another very similar rat model of absence epilepsy) [48]. The same may apply to IL6 levels; however, we decided not to measure this cytokine levels according to the knowledge that, in systemic diseases more frequently treated with TCZ (e.g., rheumatoid arthritis), it is not possible to predict the response to therapy by serum IL-6 levels, as these may not change significantly $[49,50]$. While we have no direct demonstration of TCZ antiepileptogenic and anti-absence effects through IL-6 block, this is supported by our experiments in co-administration (see "Tocilizumab Effects Against LPS and IL-6 Administration") and the knowledge that TCZ is a monoclonal antibody highly specific for this cytokine.

IL-6, a proinflammatory cytokine, is typically found in low quantities in the CNS, but stimulation of astrocytes and microglia can lead to increased production [15]. IL-6 is also upregulated by increased levels of other cytokines such as TNF- $\alpha$, IL-I $\beta$, IFN- $\gamma$, and IL-17 [17]. Some patient studies have reported an increase of IL-6 levels, plasma and CSF [8, 20, 51-54], in patients with different epileptic conditions. Thus, IL-6 is proposed to be a contributor to seizure predisposition and occurrence and seizure-related brain injury [55]. This hypothesis is further supported by experimental evidence in which IL-6 is increased in the cortex and striatum after audiogenic seizures in Wistar audiogenic rats (WAR) and that IL-6 mRNA rapidly increased in the hippocampus after status epilepticus induced by pilocarpinelithium in rats $[56,57]$.

Inflammation has not been clearly linked to absence seizures in WAG/Rij rats and, in fact, is known that cytokine brain levels do not differ significantly from non-epileptic control ACI rats [26]. However, drugs acting on inflammatory background (e.g., etoricoxib, indomethacin, fingolimod or rapamycin) decreased the epileptogenesis process in WAG/Rij rats [38, 41, 43, 44] and LPS or cytokine administration (including IL-6 as demonstrated in our experiments; see "Tocilizumab Effects Against LPS and IL-6 Administration") increased SWD activity [26, $30,31]$. These results indicate a role for neuroinflammation and therefore support TCZ effects in this model through its antiinflammatory mechanism.

On the other hand, we observed that TCZ effects are only temporary as it happens also for other drugs (e.g., fingolimod, etoricoxib, ethosuximide, and levetiracetam) and this has previously been explained by the ability of genetically determined epileptogenesis to restart at any time during animal life and the lack of efficacy of the treatments in inducing a permanent effect $[23,38,43,58]$. Furthermore, considering that TCZ is also effective against absence seizures in adult
WAG/Rij rats, this effect may contribute to the final antiepileptogenic properties demonstrated. This would also raise the hypothesis that TCZ is delaying seizure onset more than acting as a disease-modifying agent.

\section{Tocilizumab and Psychiatric Comorbidity}

Development of SWDs has previously been associated with depressive-like behavior in WAG/Rij rats [59-61]. In agreement with the hypothesis that SWDs are necessary for the development of behavioral alterations [59], we found that ELTT with TCZ significantly decreased IT in FST, at both doses, therefore displaying antidepressant-like effects, while TCZ did not affect anxiety-like behavior in the EPM test. While this effect may be secondary to TCZ inhibition of absence seizures development (in line with several other previous observations), it is not possible to exclude that TCZ antidepressant-like effects are mediated by its action on IL- 6 [24]. In fact, neuroinflammatory pathogenic mechanisms have been identified in rats with coexisting chronic epilepsy and depression and it is logical to assume that inflammation may represent a causal link between the two disorders, thereby explaining the high degree of comorbidity between epilepsy and depression $[4,5,62]$. Among the proinflammatory cytokines, preclinical and clinical studies have demonstrated that IL-6 may have a special role in the pathogenesis of depression. Preclinical studies have provided evidence on sustained elevations of IL- 6 in the CNS as a main contributor to depressivelike phenotypes in animal models [63, 64]. Transgenic mice that overexpressed IL- 6 centrally presented also an increased immobility time in the FST [64] similar to animals that received intra-cranial injections of IL-6 [65, 66]. Instead, IL-6 knockout mice showed a reduced depressive-like behavior and were more resistant to behavioral stress, suggesting a functional role for IL-6 also in the molecular mechanism of depression [67, 68]. The involvement of IL-6 in depressionlike behaviors is consistent with clinical studies in humans; increased levels of IL- 6 have been found in the plasma [69-71] and brain of patients with mood disorders [72-74].

Therefore, the mechanism by which TCZ positively influences depressive behavior in WAG/Rij might also be due to the modulation of IL-6 proinflammatory cytokine as in the case of both IL-6 antibody and soluble gp130 (a natural inhibitor of the IL-6/sIL-6R complex) blocking the effects of IL-6 infusion on immobility, improving depressive-like symptoms in mice [64]. Finally, preliminary clinical evidence assessing proinflammatory cytokines in mood disorders have demonstrated a favorable impact of anti-cytokine treatment and TCZ therapy in treatment-refractory major depression [75] and on depressive symptoms in patients with rheumatoid arthritis [76-78]. 


\section{Tocilizumab Effects Against LPS and IL-6 Administration}

Considering all the abovementioned results, it seems reasonable to suggest that our observed effects of TCZ could be mediated through inhibition of the IL-6 signaling system in the brain of WAG/Rij rats. To validate this hypothesis, we decided to test whether IL-6 would increase per se SWDs and then measure the effects of TCZ against the increased SWDs induced by IL-6. The bacterial endotoxin lipopolysaccharide (LPS) is a general stimulator of microglia to produce the proinflammatory and/or cytotoxic factors $[29,30]$.

We demonstrated for the first time that IL-6 directly injected into the brain is able to increase SWD parameters for a short time of period of about $4 \mathrm{~h}$. Pretreatment with TCZ, inhibiting IL-6 receptors, was sufficient to completely suppress the increased SWD parameters. These results indicate that the inflammatory response, particularly that mediated by IL-6 in the brain, is critically involved in absence seizure generation in WAG/Rij rats. Furthermore, these data definitively support the central effects of TCZ. This is also in line with results showing that a specific anti-inflammatory approach, which blocked IL-1 $\beta$ biosynthesis, reduces SWD activity in adult GAERS, demonstrating the likely contribution of proinflammatory cytokines to absence epilepsy [48].

In order to further study the role of inflammation and TCZ effects, we tested its efficacy in preventing LPS-induced increase in absence seizures of WAG/Rij rats; it is known that LPS administration is able to increase SWDs activity, in this and other strains, and this has been linked to an increase in peripheral and cerebral cytokine levels such as IL-6, IL-1 $\beta$ and TNF- $\alpha[30,79]$. In this regard, we found that TCZ pretreatment significantly reduced the LPS-induced increase of absence seizure in WAG/Rij rats indicating that LPSmediated effects are strongly linked to IL-6 activity. This is in agreement with previous findings indicating that LPS normally induces IL-6 in both astrocytes and microglia [80, 81].

\section{Conclusions}

Overall, our results suggest that the proinflammatory cytokine IL-6 may have a role in the generation of neuronal hyperexcitability and contributes to the modulation of absence epileptic activity in WAG/Rij rats. The interruption of the IL-6 signaling pathway through administration of TCZ, a neutralizing antibody to the IL-6R, reduced the development of absence seizures and depressive-like comorbidity in this model. As such, targeting cytokines may open the perspective to develop specific anti-inflammatory approaches for managing this nonconvulsive form of epilepsy and its related neuropsychiatric comorbidities. To more effectively design anti-IL-6 treatments for absence epilepsy, it is advantageous to understand how IL-6 signals, in concert with other inflammatory mediators, produce epileptic features. In particular, more studies are necessary to evaluate the actual clinical applicability of TCZ as an anti-epileptogenic/antiepileptic drug or as an adjuvant therapy for absence epilepsy which needs to be further confirmed by controlled studies.

Acknowledgments We would like to thank to Dr. Giovanni Bosco Politi for providing technical help. This work was partly supported by the Italian Ministry of Health, Grant No. GR-2013-02355028. This work was partly supported by the Italian Ministry of University and Research (MIUR), Prot. $\mathrm{N}^{\circ}$ 2015XSZ9A2 and Prot. $\mathrm{N}^{\circ}$ 2017YZF7MA.

Required Author Forms Disclosure forms provided by the authors are available with the online version of this article.o

\section{Compliance with Ethical Standards}

Conflict of Interest The authors declare that there are no conflicts of interest.

\section{References}

1. Marchi N, Granata T, Janigro D. Inflammatory pathways of seizure disorders. Trends Neurosci 2014; 37: 55-65.

2. Bauer J, Becker AJ, Elyaman W, et al. Innate and adaptive immunity in human epilepsies. Epilepsia 2017; 58: 57-68.

3. Devinsky O, Vezzani A, Najjar S, et al. Glia and epilepsy: Excitability and inflammation. Trends Neurosci 2013; 36: 174184

4. Paudel YN, Shaikh MF, Shah S, et al. Role of inflammation in epilepsy and neurobehavioral comorbidities: Implication for therapy. Eur J Pharmacol 2018; 837: 145-155.

5. Mazarati AM, Lewis ML, Pittman QJ. Neurobehavioral comorbidities of epilepsy: Role of inflammation. Epilepsia 2017; 58: 48-56.

6. Terrone G, Salamone A, Vezzani A. Inflammation and Epilepsy: Preclinical Findings and Potential Clinical Translation. Curr Pharm Des 2017; 23: 5569-5576.

7. van Vliet EA, Aronica E, Vezzani A, et al. Review: Neuroinflammatory pathways as treatment targets and biomarker candidates in epilepsy: emerging evidence from preclinical and clinical studies. Neuropathol Appl Neurobiol 2018; 44: 91-111.

8. Lehtimäki KA, Liimatainen $\mathrm{S}$, Peltola J, et al. The serum level of interleukin-6 in patients with intellectual disability and refractory epilepsy. Epilepsy Res 2011; 95: 184-187.

9. Vezzani A, Viviani B. Neuromodulatory properties of inflammatory cytokines and their impact on neuronal excitability. Neuropharmacology 2015; 96: 70-82.

10. Bauer ME, Teixeira AL. Inflammation in psychiatric disorders: What comes first? Annals of the New York Academy of Sciences 2019; 1437: 57-67.

11. Kappelmann N, Lewis G, Dantzer R, et al. Antidepressant activity of anti-cytokine treatment: A systematic review and meta-analysis of clinical trials of chronic inflammatory conditions. $\mathrm{Mol}$ Psychiatry 2018; 23: 335-343.

12. Berg AT, Altalib HH, Devinsky O. Psychiatric and behavioral comorbidities in epilepsy: A critical reappraisal. Epilepsia 2017; 58: 1123-1130.

13. Keezer MR, Sisodiya SM, Sander JW. Comorbidities of epilepsy: Current concepts and future perspectives. The Lancet Neurology 2016; 15: 106-115. 
14. Campbell IL, Erta M, Lim SL, et al. Trans-signaling is a dominant mechanism for the pathogenic actions of interleukin-6 in the brain. J Neurosci 2014; 34: 2503-2513.

15. Rothaug M, Becker-Pauly C, Rose-John S. The role of interleukin6 signaling in nervous tissue. Biochimica et Biophysica Acta Molecular Cell Research 2016; 1863: 1218-1227.

16. Hodes GE, Ménard C, Russo SJ. Integrating Interleukin-6 into depression diagnosis and treatment. Neurobiology of Stress 2016; 4: $15-22$.

17. Erta M, Quintana A, Hidalgo J. Interleukin-6, a major cytokine in the central nervous system. International Journal of Biological Sciences 2012; 8: 1254-1266.

18. Uludag IF, Duksal T, Tiftikcioglu BI, et al. IL-1 $\beta$, IL-6 and IL1Ra levels in temporal lobe epilepsy. Seizure 2015; 26: 22-25.

19. Liimatainen S, Fallah M, Kharazmi E, et al. Interleukin-6 levels are increased in temporal lobe epilepsy but not in extra-temporal lobe epilepsy. J Neurol 2009; 256: 796-802.

20. Peltola J, Palmio J, Korhonen L, et al. Interleukin-6 and interleukin1 receptor antagonist in cerebrospinal fluid from patients with recent tonic-clonic seizures. Epilepsy Res 2000; 41: 205-211.

21. De Simoni MG, Perego C, Ravizza T, et al. Inflammatory cytokines and related genes are induced in the rat hippocampus by limbic status epilepticus. Eur J Neurosci 2000; 12: 2623-2633.

22. Khandaker GM, Pearson RM, Zammit S, et al. Association of serum interleukin 6 and C-reactive protein in childhood with depression and psychosis in young adult life a population-based longitudinal study. JAMA Psychiatry 2014; 71: 1121-1128.

23. Russo E, Citraro R, Constanti A, et al. Upholding WAG/Rij rats as a model of absence epileptogenesis: Hidden mechanisms and a new theory on seizure development. Neurosci Biobehav Rev 2016; 71: 388-408.

24. Russo E, Citraro R. Pharmacology of epileptogenesis and related comorbidities in the WAG/Rij rat model of genetic absence epilepsy. J Neurosci Methods 2018; 310: 54-62.

25. Leo A, Citraro R, Tallarico M, et al. Cognitive impairment in the WAG/Rij rat absence model is secondary to absence seizures and depressive-like behavior. Prog Neuro-Psychopharmacology Biol Psychiatry 2019; 94: 109652.

26. Van Luijtelaar G, Lyashenko S, Vastyanov R, et al. Cytokines and absence seizures in a genetic rat model. Neurophysiology 2012; 43: 478-486.

27. Györffy B, Kovács Z, Gulyássy P, et al. Brain protein expression changes in WAG/Rij rats, a genetic rat model of absence epilepsy after peripheral lipopolysaccharide treatment. Brain Behav Immun 2014; 35: 86-95.

28. Auvin S, Shin D, Mazarati A, et al. Inflammation induced by LPS enhances epileptogenesis in immature rat and may be partially reversed by IL1RA. Epilepsia 2010; 51: 34-38.

29. Turrin NP, Gayle D, Ilyin SE, et al. Pro-inflammatory and antiinflammatory cytokine mRNA induction in the periphery and brain following intraperitoneal administration of bacterial lipopolysaccharide. Brain Res Bull 2001; 54: 443-453.

30. Russo E, Andreozzi F, Iuliano R, et al. Early molecular and behavioral response to lipopolysaccharide in the $\mathrm{WAG} / \mathrm{Rij}$ rat model of absence epilepsy and depressive-like behavior, involves interplay between AMPK, AKT/mTOR pathways and neuroinflammatory cytokine release. Brain Behav Immun 2014; 42: 157-168.

31. Kovács Z, Dobolyi Á, Juhász G, et al. Lipopolysaccharide induced increase in seizure activity in two animal models of absence epilepsy WAG/Rij and GAERS rats and Long Evans rats. Brain Res Bull 2014; 104: 7-18.

32. Kishimoto T, Kang S, Tanaka T. IL-6: A New Era for the Treatment of Autoimmune Inflammatory Diseases. In: Innovative Medicine. 2015, pp. 131-147.

33. Jun JS, Lee ST, Kim R, et al. Tocilizumab treatment for new onset refractory status epilepticus. Ann Neurol 2018; 84: 940-945.
34. Krogias C, Hoepner R, Müller A, et al. Successful treatment of anticaspr2 syndrome by interleukin 6 receptor blockade through tocilizumab. JAMA Neurol 2013; 70: 1056-1059.

35. Ding C, Jones G. Anti-Interleukin-6 Receptor Antibody Treatment in Inflammatory Autoimmune Diseases. Rev Recent Clin Trials 2008; 1: 193-200.

36. Nishimoto N, Yoshizaki K, Maeda K, et al. Toxicity, pharmacokinetics, and dose-finding study of repetitive treatment with the humanized anti-interleukin 6 receptor antibody MRA in rheumatoid arthritis. Phase I/II clinical study. J Rheumatol 2003; 30: 14261435.

37. Citraro R, Leo A, Franco V, et al. Perampanel effects in the WAG/ Rij rat model of epileptogenesis, absence epilepsy, and comorbid depressive-like behavior. Epilepsia 2017; 58: 231-238.

38. Leo A, Citraro R, Amodio N, et al. Fingolimod Exerts only Temporary Antiepileptogenic Effects but Longer-Lasting Positive Effects on Behavior in the WAG/Rij Rat Absence Epilepsy Model. Neurotherapeutics 2017; 14: 1134-1147.

39. Citraro R, Leo A, Aiello R, et al. Comparative Analysis of the Treatment of Chronic Antipsychotic Drugs on Epileptic Susceptibility in Genetically Epilepsy-prone Rats. Neurotherapeutics 2015; 12: 250-262.

40. Citraro R, Leo A, De Caro C, et al. Effects of Histone Deacetylase Inhibitors on the Development of Epilepsy and Psychiatric Comorbidity in WAG/Rij Rats. Mol Neurobiol 2020; 57: 408-421.

41. Russo E, Citraro R, Donato G, et al. MTOR inhibition modulates epileptogenesis, seizures and depressive behavior in a genetic rat model of absence epilepsy. Neuropharmacology 2013; 69: 25-36.

42. Terrone G, Balosso S, Pauletti A, et al. Inflammation and reactive oxygen species as disease modifiers in epilepsy. Neuropharmacology; 167. DOI: https://doi.org/10.1016/j. neuropharm.2019.107742.

43. Citraro R, Leo A, Marra R, et al. Antiepileptogenic effects of the selective COX-2 inhibitor etoricoxib, on the development of spontaneous absence seizures in WAG/Rij rats. Brain Res Bull 2015; 113: 1-7.

44. Rimoli MG, Russo E, Cataldi M, et al. T-type channel blocking properties and antiabsence activity of two imidazo[1,2-b] pyridazine derivatives structurally related to indomethacin. Neuropharmacology 2009; 56: 637-646.

45. Russo E, Citraro R, Scicchitano F, et al. Comparison of the antiepileptogenic effects of an early long-term treatment with ethosuximide or levetiracetam in a genetic animal model of absence epilepsy. Epilepsia 2010; 51: 1560-1569.

46. Cantarín-Extremera V, Jiménez-Legido M, Duat-Rodríguez A, et al. Tocilizumab in pediatric refractory status epilepticus and acute epilepsy: Experience in two patients. J Neuroimmunol; 340. DOI: https://doi.org/10.1016/j.jneuroim.2019.577142.

47. Gruol DL. IL-6 regulation of synaptic function in the CNS Neuropharmacology 2015; 96: 42-54.

48. Akin D, Ravizza T, Maroso M, et al. IL-1 $\beta$ is induced in reactive astrocytes in the somatosensory cortex of rats with genetic absence epilepsy at the onset of spike-and-wave discharges, and contributes to their occurrence. Neurobiol Dis 2011; 44: 259-269.

49. Choi IA, Lee SJ, Park W, et al. Effects of tocilizumab therapy on serum interleukin-33 and interleukin-6 levels in patients with rheumatoid arthritis. Arch Rheumatol 2018; 33: 389-394.

50. Vilaiyuk S, Lerkvaleekul B, Soponkanaporn S, et al. Correlations between serum interleukin 6 , serum soluble interleukin 6 receptor, and disease activity in systemic juvenile idiopathic arthritis patients treated with or without tocilizumab. Cent Eur J Immunol 2019; 44: $150-158$.

51. Peltola J, Laaksonen J, Haapala AM, et al. Indicators of inflammation after recent tonic-clonic epileptic seizures correlate with plasma interleukin-6 levels. Seizure 2002; 11: 44- 46. 
52. Lehtimäki KA, Keränen T, Palmio J, et al. Increased plasma levels of cytokines after seizures in localization-related epilepsy. Acta Neurol Scand 2007; 116: 226-230.

53. Tekgul H, Polat M, Tosun A, et al. Cerebrospinal fluid interleukin-6 levels in patients with west syndrome. Brain Dev 2006; 28: 19-23.

54. Lehtimäki KA, Keränen T, Huhtala H, et al. Regulation of IL-6 system in cerebrospinal fluid and serum compartments by seizures: The effect of seizure type and duration. J Neuroimmunol 2004; 152: 121-125.

55. de Vries EE, van den Munckhof B, Braun KPJ, et al. Inflammatory mediators in human epilepsy: A systematic review and meta-analysis. Neurosci Biobehav Rev 2016; 63: 177-190.

56. Rosell DR, Nacher J, Akama KT, et al. Spatiotemporal distribution of gp130 cytokines and their receptors after status epilepticus: Comparison with neuronal degeneration and microglial activation. Neuroscience 2003; 122: 329-348.

57. de Souza Bernardino TC, Teixeira AL, Miranda AS, et al. Wistar Audiogenic Rats (WAR) exhibit altered levels of cytokines and brain-derived neurotrophic factor following audiogenic seizures. Neurosci Lett 2015; 597: 154-158.

58. Leo A, De Caro C, Nesci V, et al. Antiepileptogenic effects of Ethosuximide and Levetiracetam in WAG/Rij rats are only temporary. Pharmacol Reports 2019; 71: 833-838.

59. Sarkisova KY, Kuznetsova GD, Kulikov MA, et al. Spike-wave discharges are necessary for the expression of behavioral depression-like symptoms. Epilepsia 2010; 51: 146-160.

60. Sarkisova K, van Luijtelaar G. The WAG/Rij strain: A genetic animal model of absence epilepsy with comorbidity of depressiony. Prog Neuro-Psychopharmacology Biol Psychiatry 2011; 35: 854 876.

61. Russo E, Citraro R, Scicchitano F, et al. Effects of early long-term treatment with antiepileptic drugs on development of seizures and depressive-like behavior in a rat genetic absence epilepsy model. Epilepsia 2011; 52: 1341-1350.

62. Mula M. Epilepsy and Psychiatric Comorbidities: Drug Selection. Current Treatment Options in Neurology; 19. DOI: https://doi.org/ 10.1007/s11940-017-0483-0.

63. Aniszewska A, Chłodzińska N, Bartkowska K, et al. The expression of interleukin- 6 and its receptor in various brain regions and their roles in exploratory behavior and stress responses. $J$ Neuroimmunol 2015; 284: 1-9.

64. Sukoff Rizzo SJ, Neal SJ, Hughes ZA, et al. Evidence for sustained elevation of IL-6 in the CNS as a key contributor of depressive-like phenotypes. Transl Psychiatry; 2. DOI: https://doi.org/10.1038/tp. 2012.120.

65. Wu TH, Lin CH. IL-6 mediated alterations on immobile behavior of rats in the forced swim test via ERK1/2 activation in specific brain regions. Behav Brain Res 2008; 193: 183-191.

66. Sakic B, Gauldie J, Denburg JA, et al. Behavioral effects of infection with IL-6 adenovector. Brain Behav Immun 2001; 15: 25-42.

67. Chourbaji S, Urani A, Inta I, et al. IL-6 knockout mice exhibit resistance to stress-induced development of depression-like behaviors. Neurobiol Dis 2006; 23: 587-594.

68. Monje FJ, Cabatic M, Divisch I, et al. Constant darkness induces IL-6-dependent depression-like behavior through the NF- $\mathrm{KB}$ signaling pathway. $J$ Neurosci 2011; 31: 9075-9083.
69. Liu Y, Ho RCM, Mak A. Interleukin (IL)-6, tumour necrosis factor alpha (TNF- $\alpha$ ) and soluble interleukin-2 receptors (sIL-2R) are elevated in patients with major depressive disorder: A metaanalysis and meta-regression. Journal of Affective Disorders 2012; 139: 230-239.

70. Howren MB, Lamkin DM, Suls J. Associations of depression with c-reactive protein, IL-1, and IL-6: A meta-analysis. Psychosom Med 2009; 71: 171-186.

71. Dowlati Y, Herrmann N, Swardfager W, et al. A Meta-Analysis of Cytokines in Major Depression. Biol Psychiatry 2010; 67: 446457.

72. Hiles SA, Baker AL, de Malmanche T, et al. A meta-analysis of differences in IL-6 and IL-10 between people with and without depression: Exploring the causes of heterogeneity. Brain, Behavior, and Immunity 2012; 26: 1180-1188.

73. Kern S, Skoog I, Börjesson-Hanson A, et al. Higher CSF interleukin- 6 and CSF interleukin- 8 in current depression in older women. Results from a population-based sample. Brain Behav Immun 2014; 41: 55-58.

74. Wang AK, Miller BJ. Meta-analysis of Cerebrospinal Fluid Cytokine and Tryptophan Catabolite Alterations in Psychiatric Patients: Comparisons between Schizophrenia, Bipolar Disorder, and Depression. Schizophr Bull 2018; 44: 75-83.

75. Adzic M, Brkic Z, Mitic M, et al. Therapeutic Strategies for Treatment of Inflammation-related Depression. Curr Neuropharmacol; 16. DOI: https://doi.org/10.2174/ $1570159 x 15666170828163048$.

76. Traki L, Rostom S, Tahiri L, et al. Responsiveness of the EuroQol EQ-5D and Hospital Anxiety and Depression Scale (HADS) in rheumatoid arthritis patients receiving tocilizumab. Clin Rheumatol 2014; 33: 1055-1060.

77. Townes S V., Furst DE, Thenkondar A. The impact of tocilizumab on physical function and quality of life in patients with rheumatoid arthritis: A systematic literature review and interpretation. Open Access Rheumatology: Research and Reviews 2012; 4: 87-92.

78. Sun Y, Wang D, Salvadore G, et al. The effects of interleukin-6 neutralizing antibodies on symptoms of depressed mood and anhedonia in patients with rheumatoid arthritis and multicentric Castleman's disease. Brain Behav Immun 2017; 66: 156-164.

79. Kovács Z, Kékesi KA, Szilágyi N, et al. Facilitation of spike-wave discharge activity by lipopolysaccharides in Wistar Albino Glaxo/ Rijswijk rats. Neuroscience 2006; 140: 731-742.

80. Woodroofe MN, Sarna GS, Wadhwa M, et al. Detection of interleukin-1 and interleukin-6 in adult rat brain, following mechanical injury, by in vivo microdialysis: evidence of a role for microglia in cytokine production. J Neuroimmunol 1991; 33: 227-236.

81. Minogue AM, Barrett JP, Lynch MA. LPS-induced release of IL-6 from glia modulates production of IL-1 $\beta$ in a JAK2-dependent manner. J Neuroinflammation; 9. DOI: https://doi.org/10.1186/ 1742-2094-9-126.

Publisher's Note Springer Nature remains neutral with regard to jurisdictional claims in published maps and institutional affiliations. 